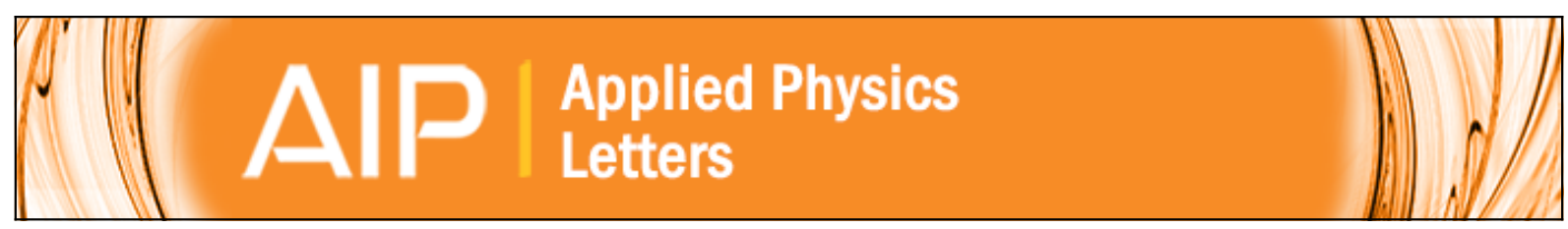

Optical metasurfaces with robust angular response on flexible substrates

Andrea Di Falco, Yang Zhao, and Andrea Alú

Citation: Applied Physics Letters 99, 163110 (2011); doi: 10.1063/1.3655332

View online: http://dx.doi.org/10.1063/1.3655332

View Table of Contents: http://scitation.aip.org/content/aip/journal/apl/99/16?ver=pdfcov

Published by the AIP Publishing

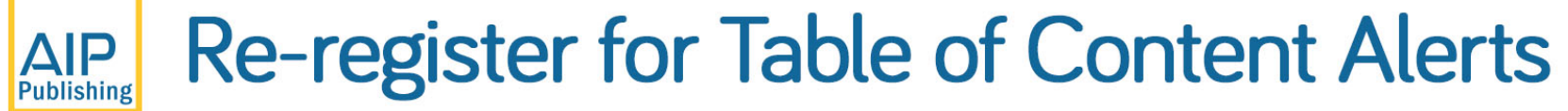

Create a profile.

Sign up today! 


\title{
Optical metasurfaces with robust angular response on flexible substrates
}

\author{
Andrea Di Falco, ${ }^{1, a)}$ Yang Zhao, ${ }^{2}$ and Andrea Alú ${ }^{2}$ \\ ${ }^{1}$ School of Physics and Astronomy, University of St. Andrews, North Haugh, St. Andrews, KY16 9SS, \\ United Kingdom \\ ${ }^{2}$ Department of Electrical and Computer Engineering, The University of Texas at Austin, Austin, Texas 78712, \\ USA
}

(Received 6 July 2011; accepted 30 September 2011; published online 21 October 2011)

\begin{abstract}
We have fabricated optical metasurfaces on flexible substrates that show an optical response independent on the polarization and angles of incidence of light. The realized devices operate as ultrathin selective filters at visible frequencies, with a bandwidth of $\sim 200 \mathrm{~nm}$, and their response is robust to membrane bending for possible integration in conformal optical devices. (C) 2011 American Institute of Physics. [doi:10.1063/1.3655332]
\end{abstract}

Metamaterials are man-made materials with engineerable bulk response, not available in any of their constituents. They may be tailored to support negative refraction ${ }^{1}$ and to implement transformation-optics rules. ${ }^{2}$ They have been proposed mostly for applications like superlensing and invisibility. $^{3,4}$ By definition, their anomalous response is based on resonant inclusions with largely sub-wavelength granularity, such that the dominant Floquet harmonic is mostly responsible for their bulk response, similar to a natural or artificial material composed of sub-wavelength atoms. Compared to photonic crystals or Bragg lattices, this property has relevant advantages in terms of bandwidth and reduced spatial dispersion effects.

Recently, it has been shown that a bulk metamaterial is not strictly necessary to obtain a negative index of refraction, ${ }^{5}$ and anomalous optical response may be obtained also from a single metasurface. The possibility to eliminate one dimension in metamaterials has strong consequences on the potential applications of this technology, because a single surface is typically easier to be fabricated and can be given additional properties, for example, flexibility, if based on polymeric substrates. The first examples of building blocks for flexible metamaterials have been demonstrated in the THz (Ref. 6) and near infrared ${ }^{7}$ range, and very recently at visible frequencies. ${ }^{8,9}$

In this context, recent interest in "metasurfaces" and "metafilms," the two-dimensional (2D) version of metamaterials, has been highlighted in several papers. ${ }^{10-12}$ Their design and characterization are in many senses consistent with the design of frequency-selective surfaces (FSSs), a common technology at microwave frequencies for filtering applications. ${ }^{13}$ FSSs are typically composed of periodic metallic inclusions or apertures on a metallic sheet with subwavelength periodicity, which provides a bulk response analogous to an averaged impedance boundary. Recently, FSS structures have been designed in the visible range, providing a response independent on the angles of incidence, ${ }^{14}$ consistent with the averaged description of such arrays. ${ }^{12,15}$

In this paper, we experimentally verify the angular independence of the optical response of plasmonic metasurfaces

\footnotetext{
a) Author to whom correspondence should be addressed. Electronic mail: adf10@st-andrews.ac.uk.
}

and apply them to the flexible substrate technology, recently developed in Ref. 9. We show numerically and experimentally that this kind of FSS implements a filter transfer function as depicted in the artistic rendering in Fig. 1. The nanopatterned membrane selectively reflects a target frequency range, independently from the polarization and the angles of incidence. The membrane virtually does not affect the other frequencies.

The fabrication procedure is outlined in Fig. 2(a). A silicon carrier is spun with a sacrificial release layer (XP-SU8 release layer, from Microchem) and baked for $1 \mathrm{~min}$ at $230{ }^{\circ} \mathrm{C}$. The sample is then spin coated with a $4 \mathrm{~mm}$ thick layer of SU8, a negative epoxy-based resist (Microchem), which is baked at $90^{\circ} \mathrm{C}$ for $5 \mathrm{~min}$, exposed at UV light for $1 \mathrm{~min}$, and baked again for $2 \mathrm{~min}$ at $100^{\circ} \mathrm{C}$ to promote and stabilize its cross-linking. This layer constitutes the membrane. A $30 \mathrm{~nm} \mathrm{Au} \mathrm{layer} \mathrm{is} \mathrm{then} \mathrm{deposited} \mathrm{on} \mathrm{the} \mathrm{sample}$ with an electron beam evaporator at low deposition rate, to ensure high film quality. The sample is then coated with a further layer of SU8, of typical thickness of $\sim 90 \mathrm{~nm}$, to be used as e-beam resist.

The pattern definition is carried out with a modified LEO-RAITH e-beam system, with a positional accuracy of $2 \mathrm{~nm}$ and a maximum acceleration voltage of $30 \mathrm{KV}$. After the exposure, the sample is baked for 2 min to complete the cross-linking and developed with ethyl lactate (EC solvent, Microchem) for $1 \mathrm{~min}$, in a gentle ultrasonic bath. The pattern is then transferred on to the gold layer with an Argon based, 4 min long reactive ion etching with a DC bias of

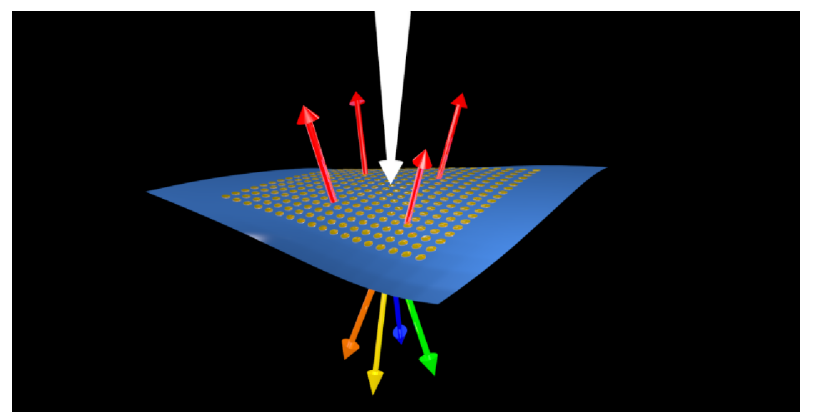

FIG. 1. (Color online) Artistic rendering of an optical FSS on flexible substrate. 
(a)

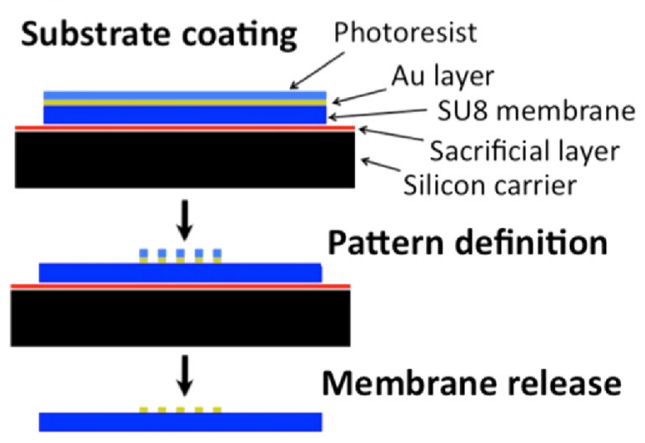

(b)

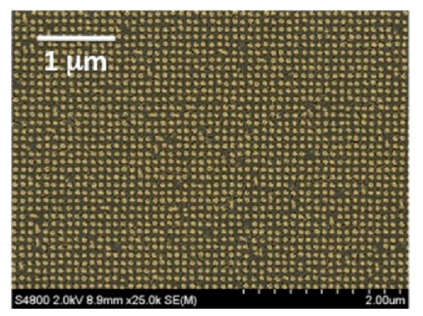

FIG. 2. (Color online) (a) Outline of the fabrication process. (b) Processed SEM picture of a typical sample.

$\sim-330 \mathrm{~V}$. The membrane is finally released from the substrate via immersion in $\mathrm{N}$-Methyl-2-pyrrolidone until complete detachment and placed on a custom built plastic frame to ease manipulation. In Fig. 2(b), we show a picture of a typical sample, taken with a scanning electron microscope (SEM), where the gold particles have been highlighted with an image processor. The target design is a two dimensional square array of gold disks of $80 \mathrm{~nm}$ diameters with a lattice constant of $100 \mathrm{~nm}$. These features are at the boundaries of the fabrication limit of our e-beam system, which results in some topological defects in the pattern, as visible in the picture. The shape of the gold inclusion is the main factor to determine the array resonance; coupling among inclusions may slightly detune the resonance frequency. The choice of circular discs and a square lattice is important to ensure isotropic response and independence on polarization.

To characterize the optical response of the filters, we used the broadband output of a halogen lamp, cascaded by a fiber collimator and a polarizer. The illumination spot on the sample had a diameter of $\sim 2 \mathrm{~mm}$, thus uniformly shining the patterned area of $200 \times 200 \mu \mathrm{m}^{2}$. The light emerging from the sample, parallel to the incident direction, was collected in transmission with a microscope objective of focal length $\mathrm{f}=13 \mathrm{~mm}$ and numerical aperture $\mathrm{NA}=0.55$, which allowed isolating the zeroth order diffraction from the metasurface array. A beam splitter allowed imaging of the sample with a camera, while the other branch of the optical setup was connected via a fiber to a silicon-based Ocean Optics spectrometer. The spectrometer limited the observed wavelength range. The sample was mounted on two rotating stages to change the polarization of the incident light (indicated with $\alpha$ in Fig. 3) and its angle of incidence ( $\theta$ in the same figure). The sample was aligned with one of the principal axis of the lattice along the axis y. The polarization angle $\alpha$ was changed from $0^{\circ}$ to $90^{\circ}$, while the incident angle $\theta$ was varied from $0^{\circ}$ to $30^{\circ}$ (this range was limited by the physical constraints given by the focal length of the objective). The

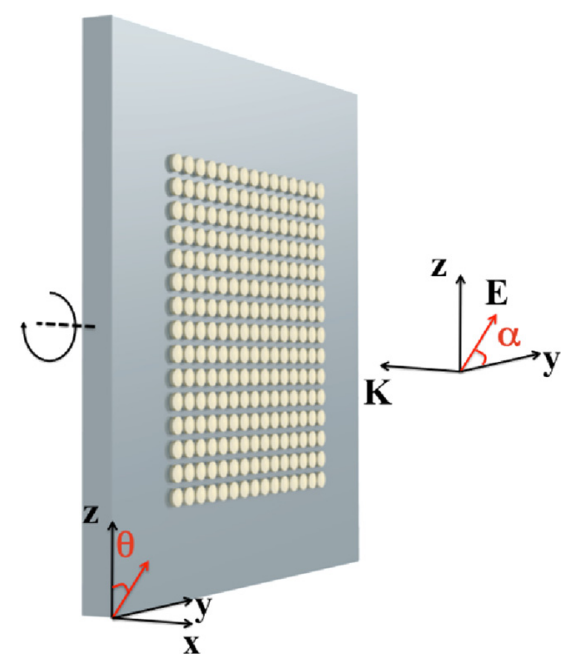

FIG. 3. (Color online) Schematic of experimental measurements. $\alpha$ is the polarization angle; $\theta$ is the incident angle.

results are summarized in Figure 4 and show a robust resonant response of the filter over a bandwidth of $\sim 200 \mathrm{~nm}$. Panel (a) shows the measured transmission curves for different incident angles and fixed polarization, whereas panel (b) shows the transmission for normal incidence and different polarization angles, respectively. Panels (c) and (d) show the corresponding full wave numerical simulation curves, obtained with software based on finite-integration. Both measured and calculated transmission curves were normalized to the transmission of a non-patterned substrate. Additionally, for the sake of clarity, the curves were numerically filtered to remove residual Fabry-Perot resonances generated at the two interfaces of the substrate.

The numerical modelling allowed for some geometrical parameter fittings: the gold film thickness and the radius of the disks were allowed to vary $\pm 2 \mathrm{~nm}$ from the nominal values; while the SU8 substrate thickness was $4.1 \mu \mathrm{m}$. The period of the gold disk in the simulation is $100 \mathrm{~nm}$. The dispersion of SU8 followed a standard 2nd order model

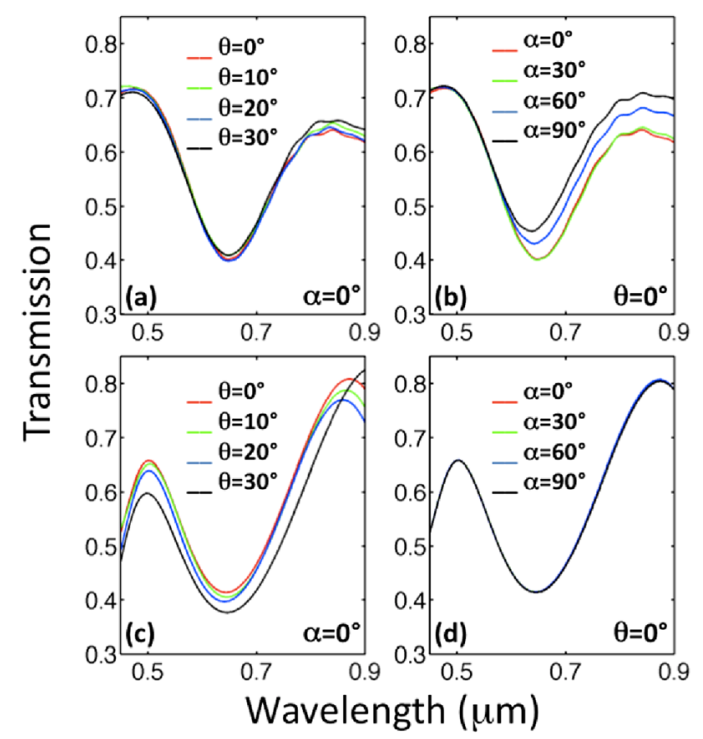

FIG. 4. (Color online) Panels (a) and (b) show the experimental transmission curves for different angles of incidence and polarization, respectively. Panels (c) and (b) are the corresponding numerical predictions. 
provided by the manufacturer. The gold permittivity was extracted from Ref. 17, with an increase of $10 \%$ in its imaginary part, uniform at all wavelengths, to take into account the random disorder in the array, as discussed in Ref. 16. Inspecting the measured curves, it is evident that the position of the dip in transmission, corresponding to the array resonance, matches perfectly with the numerical simulations. The numerical curves show an increase in transmission for longer wavelength, which does not appear in the experimental results. We believe that this is due to fabrication imperfections which limit the maximum achievable transmission. Additionally, it should be noted that in the experimental case the two orthogonal polarizations $(\alpha=0$ and $\alpha=90)$ are not degenerate. With all probability, this is due to imperfections in the shape of the gold disks and to a stretching of the membrane during its framing. This last issue would result in a rectangular lattice.

It is noticed that the resonant response of the realized filters is dependent on stretching deformations, which could be used to control their response, but it is insensitive to bending, since the resonant dip is not affected by the variations in incidence angles. To further verify this behaviour, we performed an additional set of simulations, as shown in Fig. 5. Fig. 5(a) displays the transmission and reflection coefficients from the metasurface on a flat SU8 substrate; Fig. 5(b) shows the same results for a curved SU8 substrate, with a curvature of $10^{\circ}$ per micron. As evident from the comparison of the two figures, the resonance is invariant when curving the substrate. Qualitative experimental tests (detailed experimental results will be the subject of future investigations) confirmed the predicted behaviour and pave the way to the use of the realized metasurface on a flexible substrate for conformal metamaterial applications. The independence of our simulations on substrate bending is not surprising in view of the results of Fig. 4 for a wide range of incidence angles. If the

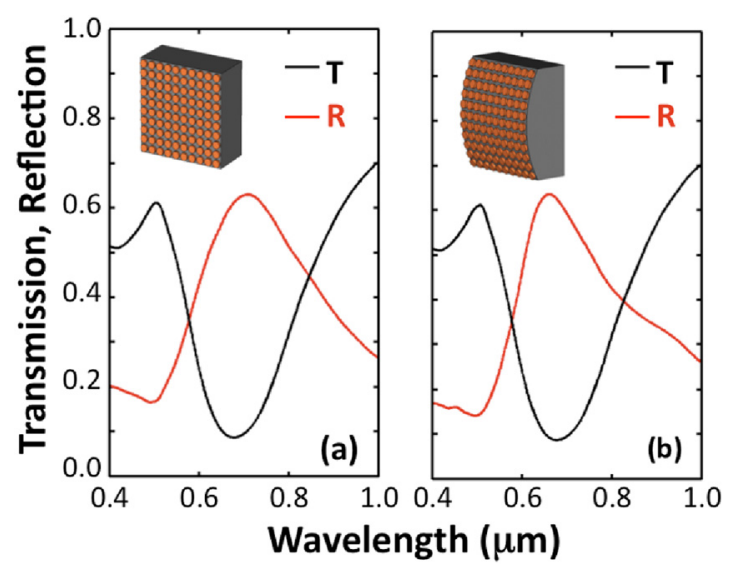

FIG. 5. (Color online) Full wave numerical simulations of (a) flat SU8 substrate and (b) curved SU8 substrate. radius of curvature of the membrane is much larger than the wavelength, bending the membrane is equivalent to locally varying the angle of incidence. For larger angular variations, or stronger bending, some minor variations of the transmission response are expected, mainly associated to the presence of losses and disorder, as theoretically discussed in Ref. 12. However, these variations are not a concern, as we expect our substrate to be mechanically bendable up to few tens of degrees per micron, which is of interest for most applications to conformal devices. For this range of curvature, the results presented here ensure the robust metasurface frequency response, independent of bending.

In conclusion, we have fabricated flexible filters implementing a frequency selective surface that has a broadband optical response independent on the angles of incidence and polarization of light, realizing the concept of flexible metasurface in the visible. These results may be applied to realize negative-index metamaterials and cloaks conformal to an object of interest. We are currently working on some of these concepts.

A.D.F. is supported by an EPSRC Career Acceleration Fellowship (EP/I004602/1). Y.Z. and A.A. were supported by an ONR MURI Grant No. N00014-10-1-0942, the NSF CAREER Award No. ECCS-0953311, and the AFOSR YIP Award No. FA9550-11-1-0009.

\footnotetext{
${ }^{1}$ V. Veselago, Physics 10, 509 (1968).

${ }^{2} \mathrm{U}$. Leonhardt and T. G. Philbin, Geometry and Light: The Science of Invisibility (Dover, Mineola, 2010).

${ }^{3}$ J. Pendry, Phys. Rev. Lett. 85, 3966 (2000).

${ }^{4}$ D. Schurig, J. J. Mock, B. J. Justice, S. A. Cummer, J. B. Pendry, A. F. Starr, and D. R. Smith, Science 314, 977 (2006).

${ }^{5}$ S. P. Burgos, R. de Waele, A. Polman, and H. A. Atwater, Nature Mater. 9, 407 (2010).

${ }^{6}$ X. G. Peralta, M. C. Wanke, C. L. Arrington, J. D. Williams, I. Brener, A. Strikwerda, R. D. Averitt, W. J. Padilla, E. Smirnova, A. J. Taylor, and J. F. O'Hara, Appl. Phys. Lett. 94, 161113 (2009).

${ }^{7}$ M. Pryce, K. Aydin, Y. A. Kelaita, R. M. Briggs, and H. A. Atwater, Nano Lett. 10, 4222 (2010).

${ }^{8}$ N. Gibbons, J. J. Baumberg, C. L. Bower, M. Kolle, and U. Steiner, Adv. Mater. 21, 3933 (2009).

${ }^{9}$ A. Di Falco, M. Ploschner, and T. F. Krauss, New J. Phys. 12, 113006 (2010).

${ }^{10}$ E. F. Kuester, M. A. Mohamed, and C. L. Holloway, IEEE Trans. Antennas Propag. 51, 2641 (2003).

${ }^{11}$ C. L. Holloway, A. Deinstfrey, E. F. Kuester, J. F. O'Hara, A. K. Azad, and A. J. Taylor, Metamaterials 3, 100 (2009).

${ }^{12}$ J. A. Gordon, C. L. Holloway, and A. Deinstfrey, IEEE Antennas Wireless Propag. Lett. 8, 1127 (2009).

${ }^{13}$ B. A. Munk, Frequency Selecetive Surfaces: Theory and Design, 1st ed. (Wiley, New York, 2000).

${ }^{14}$ D. Van Labeke, D. Gérard, B. Guizal, and F. I. Baida, Opt. Express 14, 11945 (2006).

${ }^{15}$ A. Alù and N. Engheta, "Optical wave interaction with two-dimensional arrays of plasmonic nanoparticles," in Structured Surfaces as Optical Metamaterials, edited by A. A. Maradudin (to be published).

${ }^{16}$ A. Alù and N. Engheta, New J. Phys. 12, 103015 (2010).

${ }^{17}$ P. B. Johnson and R. W. Christy, Phys. Rev. B 6, 4370 (1972).
} 